others all showed personality disorder of various kinds. Almost all had a previous history of selfmutilation, slashing of the wrists, or previous suicide attempts. The largest group, four patients, consisted of long-standing transsexuals whose disturbed personalities had possibly suggested a poor prognosis for sex reassignment surgery, and who consequently had been refused such surgery by the various specialists they had encountered. Of interest in a generally young population is the higher than average age of the group. While the high proportion of personality disorders may simply reflect the catchment population, which may also account in part for the low number of psychotic patients, this report serves to emphasise that such behaviour is not necessarily, nor particularly often, associated with paranoid schizophrenia. The most common associations seem to be a disturbance of sexual identity, a previous history of self-mutilation, and personality disorder.

G. NeIL CONACHER

Regional Treatment Centre, GeORGe H. WeSTWOOD

Kingston Penitentiary

Ontario, Canada

\section{Ethnic labels in South Africa}

SIR: We refer to the correspondence regarding the use of South African Population Registration Act categories as a basis for psychiatric research (Sashidharan \& Lipsedge, Journal, April 1986, 148, 484; Teggin et al, Journal, November 1986, 149, 667-668; Graham, Journal, November 1986, 149, 669). During the time that we were working at the MRC/University of Cape Town Clinical Psychiatry Research Unit this was a major issue of debate. The labels "Black", "Coloured", "Indian" and "White" as used in South Africa are fundamentally political, and do not refer in any scientific sense to discrete ethnic or cultural groups (Sharp, 1980). The important way in which these labels are "real" is that they dictate vastly differing access to resources of all kinds, including housing, education, employment, and health care.

Department of Psychology

BEVERLEY J. DickMAN LESLIE SWARTZ University of Cape Town Private Bag Rondebosch 7700 South Africa

\section{Reference}

SHARP, J. (1980) Can we study ethnicity? A critique of fields of study in South African anthropology. Social Dynamics, 6, 1-16.

\section{The Continuum of Psychosis and the Gene}

SIR: Crow attempts to replace the Kraepelinian dichotomy between manic depressive psychosis and schizophrenia with a continuum of psychosis (Journal, October 1986, 149, 419-429). We agree that data such as those obtained by Kendell et al support the conclusion that a symptomatic continuum exists. However, the evidence for a genetic continuum of the form envisaged by Crow is less persuasive.

Crow's model is based on several studies from the older literature reporting an excess of individuals with schizophrenia among the offspring of patients with affective disorder while failing to observe the converse. None of these studies employed modern diagnostic criteria, and it is possible that misclassification occurred. Indeed, studies (quoted by Crow) using modern methods have failed to show an increase in schizophrenia among the relatives of probands with affective disorder. Crow also argues that similarities in season of birth effects point to an underlying commonality of genetic mechanisms. However, the season of birth effect in schizophrenia is associated with an absence of a manifest genetic predisposition, suggesting a relationship with environmental factors (Boyd et al, 1986).

In contrast, evidence that schizophrenia and affective disorder are based on two independent genetic diatheses is more compelling. Firstly, as Crow acknowledges, the two major functional psychoses by and large breed true. Secondly, bipolar affective disorder appears to have a stronger genetic component than schizophrenia. Crow's hypothesis would appear to predict the converse. Thirdly, Elsässer (1952) found an equal prevalence of the two psychoses in the children of marriages between an affective and a schizophrenic individual. A greater prevalence of schizophrenia than affective disorder is predicted by Crow's hypothesis. Finally, Crow's model also fails to take into account the existence of "schizophrenia spectrum disorders", or the evidence that minor depression and depressive and cyclothymic personality disorder are genetically related to affective disorder. These findings suggest that there are separate phenotypic spectra related to the schizophrenic and the affective genotypes which are orthogonal to the phenomenological continuum that exists between the two disorders.

Given this evidence for independent genotypes, where does this leave the nosological status of schizoaffective disorder which occupies the intermediate position in the symptomatic continuum? One possibility is that it consists of both affective and schizophrenic types of illness. However, as Crow reminds us, the concept arose out of the failure 